\title{
Laboreal
}

Volume $4 \mathrm{~N}^{\circ} 1$ | 2008

Ergologia, trabalho, desenvolvimentos

\section{La dimensión emparentada del trabajo de auxiliares de enfermería a Unidad Neonatal : un análisis desde el punto de vista de l'actividad}

A dimensão relacional do trabalho de auxiliares de enfermagem em Unidade Neonatal : uma análise do ponto de vista da atividade

La dimension relationnelle du travail des aides-soignantes à Unité Neonatal :

une analyse du point de vue de l'activité

The work relational dimension of technician nursemaids within a Neonatal Care

Unit : analysis under the activity point of view

Letícia Pessoa Masson

\section{OpenEdition}

Journals

Edición electrónica

URL: http://journals.openedition.org/laboreal/11993

DOI: $10.4000 /$ laboreal. 11993

ISSN: 1646-5237

Editor

Universidade do Porto

Referencia electrónica

Letícia Pessoa Masson, « La dimensión emparentada del trabajo de auxiliares de enfermería a Unidad Neonatal : un análisis desde el punto de vista de l'actividad », Laboreal [En línea], Volume $4 N^{0} 1$ | 2008, Publicado el 01 julio 2008, consultado el 24 septiembre 2020. URL : http://journals.openedition.org/ laboreal/11993 ; DOI : https://doi.org/10.4000/laboreal.11993

Este documento fue generado automáticamente el 24 septiembre 2020

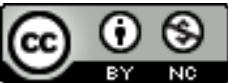

Laboreal está licenciado com uma Licença Creative Commons - Atribuição-NãoComercial 4.0 Internacional. 


\section{La dimensión emparentada del} trabajo de auxiliares de enfermería a Unidad Neonatal : un análisis desde el punto de vista de l'actividad

A dimensão relacional do trabalho de auxiliares de enfermagem em Unidade Neonatal : uma análise do ponto de vista da atividade La dimension relationnelle du travail des aides-soignantes à Unité Neonatal : une analyse du point de vue de l'activité

The work relational dimension of technician nursemaids within a Neonatal Care Unit : analysis under the activity point of view

Letícia Pessoa Masson

\section{REFERENCIA}

Masson, L. (2007) A dimensão relacional do trabalho de auxiliares de enfermagem em Unidade Neonatal : uma análise do ponto de vista da atividade. Dissertação de Mestrado em Saúde Pública. Escola Nacional de Saúde Pública/ Fundação Oswaldo Cruz, Rio de Janeiro.

\section{NOTA DEL EDITOR}

Manuscrito recibido en : abril/2008

Aceptado tras peritage en : junio/2008 


\section{Introducción}

Presentamos una pesquisa de tesis realizada con auxiliares de enfermería de una Unidad Neonatal (UN) en una materni- dad pública de Rio de Janeiro, Brasil. El estudio buscó com- prender-transformar la relación entre la (invisibilidad de la) actividad emparentada de estas trabajadoras y su salud.

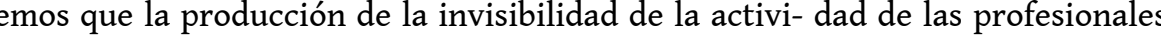
denominadas "auxiliares de enfer- mería" está vinculada especialmente a la histórica división sexual y social del trabajo en nuestra sociedad, que se cons- tituye en una norma antecedente (Schwartz, 2000) fuerte, pues asociada a la subvaloración e incluso a la valoración negativa de este colectivo profesional casi totalmente femeni- no, considerado poco escolarizado y cualificado (Scavone, 2005; Daune-Richard, 2003; Kergoat, 1996).

3 La salud es aquí considerada como capacidad normativa, con- cretizada en la recreación permanente del medio, en dirección a valores propios (Canguilhem, 2002). En este sentido, salud re- presenta la posibilidad de gestionar los riesgos, es decir, de conquistar condiciones favorables para lidiar con el sufrimiento, dándole un rumbo creativo, no patogénico. Caminamos, por lo tanto, a lo largo de una perspectiva exclusivamente basada en cuadros (psico)patológicos eventualmente encontrados.

Por actividad emparentada se entiende la preponderancia del "relacionarse" propio de los humanos, lo que encontramos en el tipo de trabajo realizado por estas profesionales, envol- viendo diversos aspectos sobrepuestos y difícilmente aisla- bles, tales como la producción charlatana, la dimensión cog- nitiva y afectivo-emocional, en fin, el envolvimiento de todo el cuerpo en la actividad (el cuerpo-si) (Schwartz \& Durrive, 2007 ; Schwartz, 2000).

\section{Objectivos}

5 La pesquisa tuvo como objetivos : i) conocer el encuadramien- to formal del trabajo de las auxiliares de enfermería de la UN estudiada (normas antecedentes y prescripciones) ; ii) aproximarse de la actividad de trabajo y constituir una Comunidad

6 Ampliada de Pesquisa ; iii) contribuir para dar visibilidad a los aspectos ocult(ad)os de la actividad de estas trabajadoras como medio de traer beneficios a la relación saludtrabajo, aliada a la calidad y productividad.

\section{Referencias Teórico-Metodológicas}

7 Teniendo como principales referencias epistemológicas la concepción canguilheniana de salud (Canguilhem, 2002) y la perspectiva ergológica (Schwartz \& Durrive, 2007), la pesqui- sa se pautó teórico-metodologicamente en planteamientos clínicos del trabajar, como la Ergonomía de la Actividad (Gué- rin et al., 2001), el Modelo Obrero Italiano de lucha por la sa- lud (Oddone et al., 1986), la Psicodinámica del Trabajo (Dejo- urs, 2004) y la Clínica de la Actividad (Clot, 2006). Utilizamos también las proposiciones y los estudios sobre la Lógica Com- petencia y la Relación de Servicio (Zarifian, 2001) y la literatu- ra relativa a las Relaciones Sociales de Sexo (Kergoat, 1996). Se visó un análisis 
de la actividad de trabajo a partir de tres herramientas metodológicas : 1. Análisis de documentos sobre las normas antecedentes/ prescripciones del trabajo, 2. Visi- tas a la UN (envolviendo observaciones de la actividad y con- versaciones informales con las auxiliares) y 3. Encuentros sobre el trabajo con las auxiliares (constituyendo el dispositi- vo Comunidad Ampliada de Pesquisa) para discusión y análi- sis de la actividad en relación con su salud, a partir del diálo- go entre el saber de la experiencia y conceptos científicos (Athayde, 2006; Brito, 2004). A través de estos instrumentos produjo el material de análisis de la pesquisa.

8 Las visitas en medio del trabajo ocurrieron en los diferentes horarios de turnos (incluso nocturnos), con vistas a recorrer las diferentes situaciones y grupos y establecer una aproxima- ción inicial con las auxiliares y su actividad de trabajo. En total fueron hechas diez visitas, en las cuales observamos la actividad y establecimos conversaciones dialogísticas con las auxiliares de enfermería, produciendo material para la etapa posterior de la pesquisa.

Tras efectuar el análisis de las normas antecedentes y de las observaciones y conversaciones mantenidas con las auxilia- res durante las visitas, fueron montados los encuentros sobre el trabajo, en los cuales discutimos con las trabajadoras te- mas pertinentes a sus demandas, en la búsqueda de un diá- logo sinérgico entre el saber científico y el saber de la prácti- ca, con vistas a comprender-transformar la actividad en foco. Fueron realizados seis encuentros de una hora, dentro de la jornada de trabajo, con la participación de nueve auxiliares de uno de los turnos (en un total de cerca de 100 auxiliares de los seis turnos), que se turnaban entre las reuniones en grupos de tres a cinco participantes. En cada encuentro hubo la presentación de los análisis y de los conceptos utilizados, colocándolos en seguida en debate con la experiencia de las trabajadoras, teniendo como foco su actividad de trabajo y la salud (en concomitancia con las exigencias de productividad y calidad). A partir de estas discusiones, se pudo problematizar/ (re) tratar cuestiones invisibilizadas/ naturalizadas (poco discutidas en el cuotidiano de trabajo) de la actividad de es- tas trabajadoras, tales como: su formación profesional, sus condiciones de trabajo, los colectivos de trabajo presentes y la organización del trabajo.

\section{Resultados}

\section{La formación profesional de las auxiliares}

10 La transmisión del saber y valores del oficio están hechas principalmente "on the job", "de auxiliar para auxiliar" y con- tribuye para la constitución y desarrollo de sus competencias. En este sentido, se reveló que la actividad de las auxiliares exige la adquisición de una "desenvoltura" y un coraje de "encarar de frente" los retos y "pasar por encima" del miedo para trabajar con los bebes en la Unidad Neonatal, envolvien- do un aprendizaje del saber no apenas en el plano cognitivo, como también en los planos corporal y afectivo.

Por otro lado, encontramos entre las auxiliares una sobrecua- lificación formal (a través de la realización de cursos de gra- duación en enfermería e incluso posgraduados, por muchas de ellas), no reconocida en ningún plano, pero que es, sin embargo, utilizada en el ejercicio de la actividad, a partir de diversas exigencias de la misma organización del trabajo. Este dato nos parece relacionado con el hecho de que las participantes 
presentan una fuerte demanda por el aprendiza- je y la incorporación de un patrimonio teórico/científico que abarque sus prácticas : ven esto como medio de no trabajar "en el automático", y de poder agregar autonomía (y quizás más valor/ reconocimiento social) a sus acciones.

\section{La construcción de colectivos en el trabajo de las auxiliares}

Identificamos la construcción de un colectivo de trabajo de las auxiliares, que posibilita relaciones de cooperación y co- ordinación entre ellas. Notamos que esta cooperación apare- ce de forma más intensa en las situaciones en que ellas con- quistaron una relativa autonomía y parece perjudicada cuando esta autonomía está impedida. Mientras, entendemos que este colectivo se presenta un tanto aislado en la interrelación con los demás grupos que actúan en la UN. Un ejemplo de eso aparece en la relación conflictiva presente en el colectivo for- mado con los familiares de los bebes para la coproducción del servicio, donde ellos son invitados (e incluso "obligado") a participar de la realización del cuidado de los hijos. En este caso, identificamos una falta de reconocimiento en doble vía : por un lado, las madres / los familiares muchas veces deman- dan de las auxiliares que estas "sientan el sufrimiento igual" al de ellas y no reconocen / entienden el empeño de las auxi- liares en realizar un trabajo con productividad y calidad, ni dan señales de reconocimiento de la importancia de la activi- dad de estas profesionales para el tratamiento efectivo de sus hijos; por otro lado, las profesionales tienen dificultades en confrontarse con las inseguridades de las madres y mismo de reconocer su sufrimiento, sus demandas afectivas, dudas, miedos y angustias frente a la situación crítica e inédita del hijo. Conforme Dejours (1993), la dinámica del reconocimiento se presenta como un medio de propiciar efectividad social al trabajo realizado y de retribución psicológica frente a la iden- tidad en el mundo del trabajo. Se debe pautar en dos senti- dos: en el de gratitud por la aportación a la organización del trabajo ; y en el de constatación de la aportación de los suje- tos para la organización del trabajo y, así, de toma de con- ciencia de las insuficiencias y fallos del proceso técnico y de la concepción de la organización del trabajo.

\section{La organización real del trabajo de las auxiliares}

13 A través de la construcción histórica de un colectivo de oficio, fueron creadas reglas profesionales para el desarrollo de las actividades frente a las insuficiencias de la organización pres- crita y de las condiciones de trabajo. En la invención de reglas (en el interior de las cuales bricolages, como "chupetes" he- chos de guantes, "rollito" de manta y adaptaciones de mate- riales), algunas presentan condiciones relativas de visibilidad entre las propias auxiliares y posibilitan la trasgresión colec- tiva de las normas antecedentes (Schwartz \& Durrive, 2007) del trabajo, en consonancia con los valores del oficio de estas trabajadoras.

Destacamos la construcción de un patrimonio del saber y va- lores ("hacer siempre lo mejor" / buscar la "perfección"; tener la salud del niño en primer lugar; ser "peleonas" por mejores condiciones y formas de organización del trabajo ; tener "corazón de madre"), formando y movilizando un género profe- sional (Clot, 2006) de las auxiliares, que se desarrolla y se trasmite entre ellas, pasando por juicio en su colectivo, lo que contribuye para la protección de la salud y para la realización de un trabajo con la calidad y la productividad pertinentes. Por parte de la jerarquía verificamos 
insuficiente (re)conoci- miento / evaluación del trabajo, lo que queda claro por la fragilidad de los entrenamientos recibidos y por la mala cali- dad de los materiales proveídos.

\section{La mala calidad de los instrumentos de trabajo y el riesgo "subjetivo" a la salud de las auxiliares}

Quedó patente a lo largo de la pesquisa la falta de condicio- nes para la gestión de los riesgos del trabajo emparentado de cuidado. Las malas condiciones de los instrumentos de traba- jo ofrecidos (ex: jelcos y jeringuillas de mala calidad, incuba- doras sin mantenimiento), a pesar de presentarse como con- diciones simplemente materiales parecen representar claramente lo cuanto el trabajo de las auxiliares es invisibili- zado y moviliza un fuerte componente afectivo para efectivar- se. Tales condiciones precarias exigen inventividad e improvi- sación continuadas - lo que viabiliza (precariamente) la efectivación del trabajo de cuidado, pero, contradictoriamen- te, contribuye para el mantenimiento de malas condiciones. En este sentido, se hieren los valores y normas del colectivo de las auxiliares, generando problemas tanto en la salud de los bebes (más perforados de lo que es necesario con jelco malo, por ejemplo), como de las auxiliares (que quedan "mal emocionalmente", sienten en si el dolor de los niños, quedan "cansadas", "estresadas", no quedan "bien de la cabeza".

\section{Consideraciones Finales}

Entendemos que las competencias necesarias para la realiza- ción del trabajo de cuidado en la UN transcurren de una mo- vilización sinérgica de conocimientos teóricotécnicos estruc- turados (los cuales las trabajadoras demandan) y del uso de la inteligencia de la práctica (Dejours, 1997), orientada hacia la dimensión emparentada de la actividad. Sin embargo, esta última, aunque fundamental en el trabajo de las auxiliares, es invisibilizada y desvalorizada en cuanto competencia desarro- llada en el cotidiano de las trabajadoras. Al contrario, al con- tarse con la movilización del cuerposi para el pleno desem- peño de la actividad de las auxiliares, eso ocurre de forma naturalizada, desconsiderándose los procesos que llevaron al desarrollo de esta competencia y los costes para la salud in- volucrados en esta movilización. Notamos también que la ac- tividad relacional queda invisibilizada por la intensidad con la cual las trabajadoras se envuelven emocionalmente con el trabajo de cuidado, dando todo de si para que los resultados sean alcanzados, lo que creemos estar fuertemente asociado a la división sexual del trabajo (considerada una importante norma antecedente), en que el "cuidar" es atribuido a las mujeres. De forma paradojal, este empeño dificulta la trans- formación de las condiciones que precarizan su actividad y perjudican su salud, en la medida en que oculta las dificulta- des existentes. De esta forma, lo que es visible es lo que deja de ser hecho, la ausencia de una tarea realizada y no los es- fuerzos emprendidos en su realización.

17 Creemos que la postura del colectivo de auxiliares frente a las cuestiones explicitadas arriba puede estar representando una forma (aunque precaria) de defensa de la salud, a través de un sistema defensivo de tipo ideológico (Athayde, 1996), lo cual se refleja en el relativo aislamiento presentado por este grupo frente a los demás que actúan en la UN. 


\section{BIBLIOGRAFÍA}

Athayde, M. (1996). Gestão de Coletivos de Trabalho e Modernidade : Questões para a Engenharia de Produção. Tese de Doutorado, CO- PPE/ Universidade Federal do Rio de Janeiro, Rio de Janeiro.

Athayde, M. (2006). Uma proposta de orientação epistemológica, teóri- ca e metodológica para a pesquisa-intervenção no campo do traba- lho humano do ponto de vista da atividade e da dialogia. Texto para discussão, Programa de Pós-Graduação em Psicologia Social/UERJ, Rio de Janeiro, (inédito).

Brito, J. (2004). Saúde do trabalhador : reflexões a partir da abordagem ergológica. In M. Figueiredo, M. Athayde, J. Brito \& D. Alvarez (Orgs.). Labirintos do trabalho : interrogações e olhares sobre o trabalho vivo (pp. 91-114). Rio de Janeiro : DP\&A.

Canguilhem, G. (2002). O normal e o patológico. Rio de Janeiro : Forense Universitária.

Clot, Y. (2006). A função psicológica do trabalho. Petrópolis : Editora Vozes.

Daune-Richard, A. M. (2003). Qualificações e representações sociais. In H. Hirata \& M. Maruani (Orgs.). As novas fronteiras da desigualda- de : homens e mulheres no mercado de trabalho. São Paulo : Editora SENAC. (pp. 65-76).

Dejours, C. (1993). Coopération et construction de l'identité en situa- tion de travail. Futur antérieur, 16, 2, 41-52. Retirado em Janeiro, 02, 2007, de http://multitudes.samizdat.net/ Cooperation-et-cons- truction-de-l.html.

Dejours, C. (1997). O fator humano. Rio de Janeiro : Editora Fundação Getúlio Vargas.

Dejours, C. (2004). Adendum - Da Psicopatologia à Psicodinâmica do Trabalho. In S. Lancman \& S. Laerte (Orgs.). Christophe Dejours : Da psicopatologia à psicodinâmica do trabalho. Rio de Janeiro : Editora Fiocruz, Brasília : Paralelo 15. (pp. 47-104).

Guérin, F., Laville, A., Daniellou, F., Durrafoug, J., \& Kerguelen, A. (2001). Compreender o Trabalho para Transformá-lo. A Prática da Ergono- mia. São Paulo : Editora Edgard Blücher Ltda.

Kergoat, D. (1996). Relações sociais de sexo e divisão sexual do traba- lho. In M. J. Lopes, D. Meyer \& V. Waldow (Orgs.). Gênero e saúde. Porto Alegre : Artes Médicas. (p 19-28).

Oddone, I., Marri, G., Glória, S., Briante, G., Chiatella, M., \& Re, A. (1986). Ambiente de trabalho : a luta dos trabalhadores pela saúde. São Paulo : Editora Hucitec.

Scavone, L. (2005). O trabalho das mulheres pela saúde : cuidar, curar, agir. In W. Villela \& S. Monteiro (Orgs.) Gênero e saúde : Programa de Saúde da Família em questão. São Paulo : ABRASCO/UNFPA/Ar- breit Factor. (p. 99-109).

Schwartz, Y. (2000). Trabalho e uso de si. Pro-posições, 11, 32, 34-50. Schwartz, Y., \& Durrive, L. (Orgs.). (2007). Trabalho e Ergologia : conversas sobre a atividade humana. Niterói : Eduff.

Zarifian, P. (2001). Objetivo Competência : por uma nova lógica. São Paulo : Atlas. 
AUTOR

\section{LETÍCIA PESSOA MASSON}

Rua João Alfredo, 37/ 101, Tijuca, Rio de Janeiro/ RJ, CEP : 20511-390, Brasil

leticiapessoa@yahoo.com.br 